\section{R. Kedia ${ }^{1}$ \\ e-mail: rajesh@irt.arco.ccm

\author{
M. L. Hunt \\ e-mail: hur @caltech.edu \\ Metr. ASME
}

\section{T. Colonius \\ e-mail: ccionius@caltech.edu}

Divisiori of Enguneering and Applied Science, Cali"arnia Ins:itute of Technology.

Pasaderia, CA 91125

\title{
Transition of Chaotic Flow in a Radially Heated Taylor-Couette System
}

Numerical simulations have been performed to study the stability of heated, incompressible Taylor-Couette flow for a radius ratio of 0.7 and a Prandtl number of 0.7 . As $G r$ is increased, the Taylor cell that has the same direction of cinculation as the natural convection current increases in size and the counterrotating cell becomes smaller. The flow remains axisymmetric and the average heat transfer decreases with the increase in Gr. When the cylinder is impulsively heated, the counterrotating cell vanishes and $n=1$ spiral is formed for $\mathrm{Gr}=1000$. This transition marks an increase in the heat transfer due to an increase in the radial velocity component of the fluid. By slowly varying the Grashof number, the simulations demonstrate the existence of a hysteresis loop. Two different stable states with same heat transfer are found to exist at the same Grashof number. A time-delay analysis of the radial velocity and the local heat transfer coefficient time is performed to determine the dimension at two Grashof numbers. For a fixed Reynolds number of 100, the two-dimensional projection of the reconstructed atrractor show's a limit cycle for $G r=-1700$. The limit cycle behavior disappears at $G r=-2100$, and the reconstructed attractor becomes irregular. The attractor dimension increases to about 3.2 from a value of 1 for the limit cycle case; similar values were determined for borh the local heat transfer and the local radial velocity, indicating that the dynamics of the temperature variations can be inferred from that of the velocity variations.

\section{Introduction}

Taylor-Couette flow (Taylor, 1923) is an interesting nontinear, dissipative dynamical system. The inner rotating cylinder provides a driving force, and as its rotation rate is increased, a series of transitions and bifurcations occur, starting with stable Taylor vortices, and leading to fully developed turbulent flow. These transitions have been widely explored (e.g., the review by Koschmieder, 1993) as a function of Reynolds number. However, buoyancy effects caused by the imposition of a radial temperature gradient cause important changes to the transitions (Kcdia et al., 1998). These are important to a number of practical devices such as high-speed pumps (Narabayashi et al., 1993).

It is useful to review the transitions for an unheated flow, such as found in the experiments of Brandstater and Swinney (1987). They considered Taylor-Couette flow with a radius ratio of 0.875 and an aspect ratio of 20 . On starting the rotation of the inner cylinder, the first flow encountered is circular steady Couette flow. The only nonzero velocity is the azimuthal velocity, which has a radial dependence. Upon increasing the Reynolds number $(\mathbf{R}=$ $\left.\omega r_{i} b / \nu\right)$, the next transition is to Taylor vortex flow (TVF) at a critical Reynolds number $\left(R_{r}=118.4\right)$. The TVF arises from a symmetrical supercritical steady bifurcation of the base flow. The flow remains time independent but with a periodic disturbance in the axial direction. L'pon further increasing the Reynolds number, another transition is made to Wavy vortex flow (WVF) at approximately $R / R_{r}=1.3$. The WVF is a time-periodic supercritical bifurcation (a Hopf bifurcation) of the TVF characterized by unsteady travelling waves. This is a first time-dependent solution with breaking of the azimuthal symmetry. Since the trajectory is a limit cycle in phase space, the corresponding attractor dimension

\footnotetext{
'Presently at ARCO Exploration and Production Technology, Plano, TX 75075.

Contributed by the Heat Transier IDivision for publication in the JOURNAL OF HEAT TKNNSFER and presented at ' 98 IMECE, Anaheim. Manuscript received by the Heat Transfer Division, July 7, 1998; revision received, Mar. 26, 1999. Keywords: Annulat Flow, Computational, Heat Transfer, Instability, Mixed Convection. Associate Technical Editor: D. Zemmbrunnen.
}

(the dimension is roughly the number of independent variables needed to model the underlying phenomenon in a dynamical system) is 1. A power spectrum would show a fundamental frequency corresponding to the azimuthal wave number and its harmonics. As the Rcynolds number is increased furthcr, modulated wavy vortex flow (MWVF) is formed at $R / R_{:}=10$. This is doubly periodic with an attractor dimension of 2 ; hence, there are two incommensurate frequencies, and the phase space portrait is a torus. At higher Reynolds number $\left(R / R_{r}=11.7\right)$, the flow becomes chaotic or "weakly turbulent." Brandstater and Swinney calculated the fractal dimension as 2.4 for $R / R_{r}=12.4$. Thereafter, with a continuous increase of Reynolds number, the flow becomes "fully turbulent" and the fractal dimension continues to increase monotonically.

The present work explores the effect of a radial temperature gradient quantified by the Grashof number $\left(\mathrm{Gr}=g \beta\left(T_{1}-\right.\right.$ $\left.\left.T_{2}\right) b^{3} / \nu^{2}\right)$ and the acceleration ratio $\left(A=\omega \omega^{2} r_{i} / g\right)$. An carlier study by Kedia, Hunt, and Colonius (1998) presented local and average heat transfer cocfficients for a fixed Reynolds number $(R=100)$ and for a range of Grashor numbers. The study alse discussed previous investigations of heated Taylor-Couette flows. Based on the heat transfer results, the flow was found to undergo a series of transitions that depend on the direction and magnitude of the imposed temperature gradient. The current work presents more detailed results on the evolution of the flow structures as the Grashof number increases, and indicates the coupling between the variations in the heat transfer rates and the changes in the sizc and shape of the Taylor vortices. The relation between the average wall shear stress and mean equivalent conductivity are presented in terms of the Colburn analogy. The mean equivalent conductivity, $K_{\text {eq }}$, is defined as the ratio of the average convective heat transfer coefficient $\left(h_{\bar{i}, z, t}\right)$ to the heat transfer coefficient for pure conduction. In addition, local measurements of the radial velocity component and the heat transfer coefficient are used to characterize the flow in terms of the attractor dimension at Iwo different Grashot numbers. 


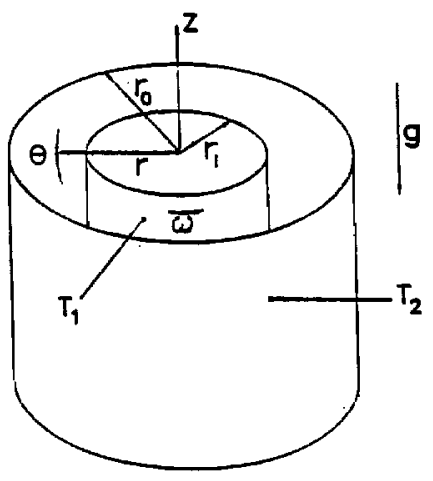

Fig. 1 Flow geometry

\section{Numerical Method}

A sketch of the flow configuration in $(r, \theta, z)$ cylindrical coordinates is shown in Fig. 1. The radii of the inner and the outer cylinders are $r_{i}$ and $r_{n}$, respectively, and the radius ratio is $\eta$. The inner cylinder rotates with a constant angular velocity $\omega$ about the vertical $z$-axis while the outer cylinder is stationary. The two cylinders are at different uniform temperatures. The temperature of the inner cylinder is $T_{1}$ and that of the outer one is $T_{2}$. Gravity acts in the negative $z$-direction. The centrifugal force is parallel to the mean temperature gradient. The temperature difference can be assumed to be sufficiently small such that the density is treated as a constant everywhere in the Navier-Stokes equations with the exception of the gravitational ( $z$-momentum equation) and the centrifugal ( $r$-momentum equation) terms. All other fluid properties are assumed to be independent of temperature. The flow is axially periodic (i.e., infinite aspect ratio) and no slip boundary conditions are used at the inner and the outer cylinders. Without heating, the Taylor cells are of uniform size. The axial distance between a pair of Taylor cells is defined as axial wavelength $(\lambda)$ and $b$ is the gap wioth. The axial wavelength is normalized by the gap width to define $L_{z}$.

The study by Kedia, Hunt and Colonius (1998) outlines the solution to the three-dimensional incompressible equations of motion with the energy equation using a Chebyshev/Fourier spectral method. Viscous terms are integrated implicitly with a CrankNicholson scheme, while the convective terms are integrated explicitly with an Adams-Bashforth scheme. The results are calculated on a $32^{3}$ mesh and dealiased with the $\frac{3}{2}$ rule. To verify the adequacy of the $32^{3}$ simulations, a series of higher resolution simulations $\left(64^{3}\right)$ with a smaller time step were also performed. The three components of velocity and the temperature were monitored at the same physical location as for the coarser resolution. The two gave an identical time trace which proved the sufficiency of the coarser simulation. The real time requirement is $13.5 \mathrm{~s}$ per time step on a SGI Onyx (IP21 processor) for a typical $32^{3}$ simulation.

The nondimensional equations and boundary conditions are given below, and follow from the earlier study by Kedia et al. (1998).

\section{Continuity:}

$$
\frac{\partial u_{r}}{\partial r}+\frac{u_{r}}{r}+\frac{1 \partial u_{\theta}}{r \partial \theta}+\frac{\partial u_{z}}{\partial z}=0
$$

\section{Momentum:}

$$
\begin{aligned}
\frac{\partial u_{r}}{\partial t}+ & \frac{u_{\theta}}{r} \frac{\partial u_{r}}{\partial \theta}-u_{z} \frac{\partial u_{r}}{\partial z}-u_{z} \frac{\partial u_{z}}{\partial r}-u_{\theta} \frac{\partial u_{\theta}}{\partial r} \\
& -\left(1-\beta^{*}\left(T-\overline{T_{u}}\right)\right) \frac{u_{\theta}^{2}}{r}=-\frac{\partial P}{\partial r} \\
& +\frac{1}{R}\left(\begin{array}{llllll}
\frac{1 \partial^{2} u_{r}}{r^{2} \partial \theta^{2}} & 1 \partial u_{\theta} & 1 \partial^{2} u_{\theta} & \partial^{2} u_{r} & \partial^{2} u_{z} \\
r^{2} \partial \theta & r \partial \theta \partial r & \partial z^{2} & \partial r \partial z
\end{array}\right)
\end{aligned}
$$

\section{Nomenclature}

$A=$ acceleration ratio, $\omega^{2} r_{i} / g$

$b=$ gap width, $\left(r_{o}-r_{i}\right)$

$C_{f}=$ friction factor, $\tau_{w} I_{\bar{\theta}, \bar{z}, \bar{r}} / \frac{1}{2} \rho\left(\omega r_{i}\right)^{2}$

$C_{p}=$ heat capacity at constant pressure

$D_{\mathrm{I}}=$ pointwise dimension

$D_{2}=$ correlation dimension

$f=$ frequency of heat transfer coefficient fluctuation

$f_{c}=$ inner cylinder frequency

$f_{s p}=$ normalized fundamental frequency for $n=2$ spiral flow

$\mathrm{Gr}=$ Grashof number, $g \beta\left(T_{1}-\right.$ $\left.T_{2}\right) b^{3} / \nu^{2}$

$g=$ acceleration due to gravity

$h=$ local heat transfer coefficient, $q /\left(T_{b}-T_{2}\right)$

$h_{\bar{\theta}, \bar{z}, t}=$ spatially and temporally averaged heat transfer coefficient

$I(\tau)=$ mutual information function

$j_{n}=$ Colburn factor

$K_{e q}=$ mean equivalent conductivity, $r_{i}$

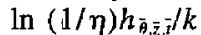

$k=$ thermal conductivity of the fluid,

$L_{z}=$ normalized axial wavelength, $\lambda / b$

$m=$ embedding dimension

$N(\epsilon)=$ number of points in a hypersphere of radius $\epsilon$ $n=$ number of starts, $2 \pi r_{o} \tan$ $\Psi / L_{\text {z }}$

$P=$ nondimensional dynamic pressurc, nondimensionalized by $\omega^{2} r_{i}^{2}$

Pr $=$ Prandtl number, $\nu / \alpha$

$P(X), P(Y)=$ probability density

$P(X, Y)=$ joint probability density

$q=$ heat flux on the outer cylinder

$\mathrm{R}=$ Reynolds number, $\omega r_{i} b / \nu$

$\mathbf{R}_{c}=$ critical Reynolds number for the onset of Taylor vortex flow

$r, \theta, z=$ cylindrical coordinates

$r_{i}=$ inner cylinder radius

$r_{0}=$ outer cylinder radius

$\mathrm{St}=$ Stanton number, $h_{\bar{\theta}, \bar{z}, \bar{r}} l$ $\rho\left(\omega r_{i}\right) C_{b}$

$T=$ nondimensional temperature, nondimensionalized by $T_{1}-T_{2}$ after subtracting $T_{2}$

$T_{1}=$ inner cylinder temperature

$T_{2}=$ outer cylinder temperature

$\underline{T_{b}}=$ bulk temperature

$\frac{T_{b}}{T_{n}}=$ dimensionless reference temperature $\begin{aligned} t & =\text { time } \\ u_{1}, u_{\theta}, u_{z} & =\text { nondimensional velocity }\end{aligned}$ component in cylindrical coordinate system, nondimensionalized by $\omega r_{i}$

$v(t)=$ time series

$\omega=$ inner cylinder angular velocity

$\rho=$ density of the fluid

$v=$ kinematic viscosity of the fluid

$\alpha=$ thermal diffusivity of the fluid

$\beta=$ thermal expansion cocfficient of the fluid

$\beta^{*}=\beta\left(T_{1}-T_{2}\right)$

$\mu=$ dynamic viscosity of the fluid

$\lambda=$ wavelength of a pair of vortices

$\eta=$ radius ratio, $r_{i} / r_{o}$

$\tau=$ delay time

$\left.\tau_{\mathcal{H}} \cdot\right|_{\dot{\theta}, \bar{i}, t}=$ spatially and temporally averaged wall shear stress

$\Psi=$ the angle of inclination of the cell from the horizontal 


$$
\begin{aligned}
\frac{\partial u_{\theta}}{\partial t}+\frac{u_{r} u_{\theta}}{r}+u_{r} \frac{\partial u_{\theta}}{\partial r}-\frac{u_{r}}{r} \frac{\partial u_{r}}{\partial \theta} & -\frac{u_{z}}{r} \frac{\partial u_{z}}{\partial \theta}+u_{z} \frac{\partial u_{\theta}}{\partial z} \\
=-\frac{1}{r} \frac{\partial P}{\partial \theta}+\frac{1}{R}\left(\frac{1}{r} \frac{\partial u_{\theta}}{\partial r}-\right. & \frac{u_{\theta}}{r^{2}}+\frac{\partial^{2} u_{\theta}}{\partial r^{2}}+\frac{1}{r^{2}} \frac{\partial u_{r}}{\partial \theta} \\
& \left.-\frac{1}{r} \frac{\partial^{2} u_{r}}{\partial r \partial \theta}-\frac{1}{r} \frac{\partial^{2} u_{z}}{\partial z \partial \theta}+\frac{\partial^{2} u_{\theta}}{\partial z^{2}}\right)
\end{aligned}
$$

$$
\begin{aligned}
\frac{\partial u_{z}}{\partial t}-u_{r} \frac{\partial u_{r}}{\partial z}+u_{r} \frac{\partial u_{z}}{\partial r}+\frac{u_{\theta}}{r} \frac{\partial u_{z}}{\partial \theta}-u_{\theta} \frac{\partial u_{\theta}}{\partial z} & =-\frac{\partial P}{\partial z} \\
+\frac{1}{R}\left(\frac{1}{r} \frac{\partial u_{z}}{\partial r}-\frac{1}{r} \frac{\partial u_{r}}{\partial z}-\frac{\partial^{2} u_{r}}{\partial r \partial z}+\frac{\partial^{2} u_{z}}{\partial r^{2}}+\right. & \left.\frac{1}{r^{2}} \frac{\partial^{2} u_{z}}{\partial \theta^{2}}-\frac{1}{r} \frac{\partial^{2} u_{\theta}}{\partial z \partial \theta}\right) \\
& +\frac{\mathrm{Gr}}{\mathrm{Re}^{2}}\left(T-\overline{T_{o}}\right)
\end{aligned}
$$
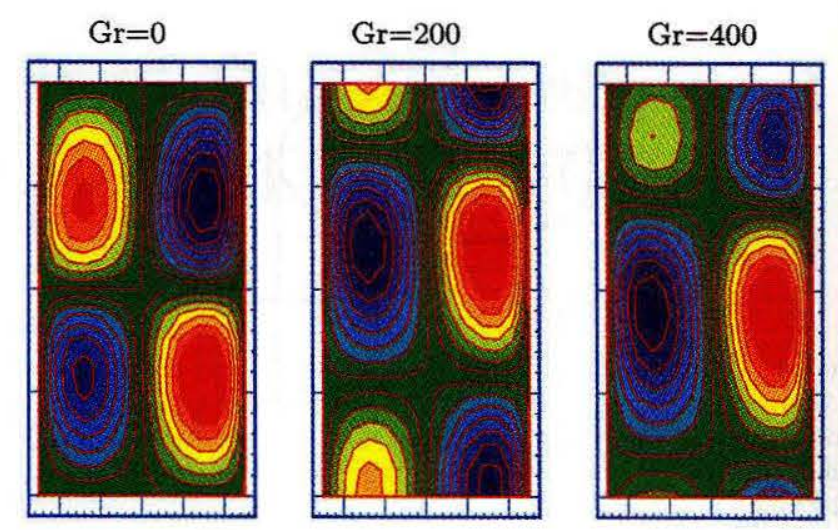

with $\left(u_{r}, u_{\theta}, u_{z}\right)=(0,1,0)$ at the nondimensional inner radius, $\eta /(1-\eta)$ and $\left(u_{r}, u_{\theta}, u_{2}\right)=(0,0,0)$ at the nondimensional outer radius, $1 /(1-\eta)$.

\section{Energy:}

$$
\begin{aligned}
\frac{\partial T}{\partial t}+u_{r} \frac{\partial T}{\partial r}+\frac{u_{\theta}}{r} \frac{\partial T}{\partial \theta}+u_{z} \frac{\partial T}{\partial z} \\
=\frac{1}{\operatorname{PrR}}\left(\frac{1}{r} \frac{\partial T}{\partial r}+\frac{\partial^{2} T}{\partial r^{2}}+\frac{1}{r^{2}} \frac{\partial^{2} T}{\partial \theta^{2}}+\frac{\partial^{2} T}{\partial z^{2}}\right)
\end{aligned}
$$

with $T=1$ at the nondimensional inner radius, $\eta /(1-\eta)$ and $T=$ 0 at the nondimensional outer radius, $1 /(1-\eta)$.

The code was validated by comparing the critical Reynolds number and the corresponding wave speeds with a linear stability analysis that included the effects of radial heating on the centrifugal and gravitational potentials (Chen and Kuo, 1990). In addition, the simulations were also compared with the results of Kataoka et al. (1977) for the local mass transfer coefficient in the limit of small Grashof number, and with the average heat transfer coefficients from the experiments of Ball et al. (1989) for a range of Grashof numbers. Further validations can also be found in Kedia (1997).

It is well known that the axial wavelength depends on Gr. At the outset, it was unclear whether the flow states ( $n=1$ spiral, $n=$ 2 spiral, or aperiodic) would be sensitive to the imposed axial wavelength, $L_{z}$. However, the numerical experiments showed that the flow states were invariant as $L_{z}$ was varied by as much as 30 percent. As $L_{z}$ was varied, the size of the cells were proportionally increased. In the absence of any published results on the exact axial wavelength for nonzero Grashof number and the robustness of the flow states with regard to $L_{z}$, the wavelength for heated flow was chosen as the wavelength at zero Grashof number (widely available from both linear stability analysis and experimental results).

\section{Results}

Flow Transitions and Hysteresis. The variation of the size of the Taylor cells (for a fixed axial wavelength) with the increase in the value of $\mathrm{Gr}$ is shown in Fig. 2. The axial velocity contours (there are two circular contours in the radial direction which corresponds to one Taylor cell) are plotted in the $r-z$ plane for $\eta=$ $0.7, L_{z}=2.001, A=0.67, \mathrm{R}=100$ and $\mathrm{Pr}=0.7$. Note that the critical Reynolds number for the onset of isothermal Taylor cells for a radius ratio of 0.7 is 79.49 . The figure illustrates two important phenomena. The first is the change in the size of Taylor cells and the second is the formation of $n=1$ spiral flow. The spirals move an axial distance of $n L_{z}$ for one rotation around the inner cylinder (Kedia et al., 1998). For $\mathrm{Gr}=0$, there is no effect of natural convection, and both the Taylor cells are of equal size. As
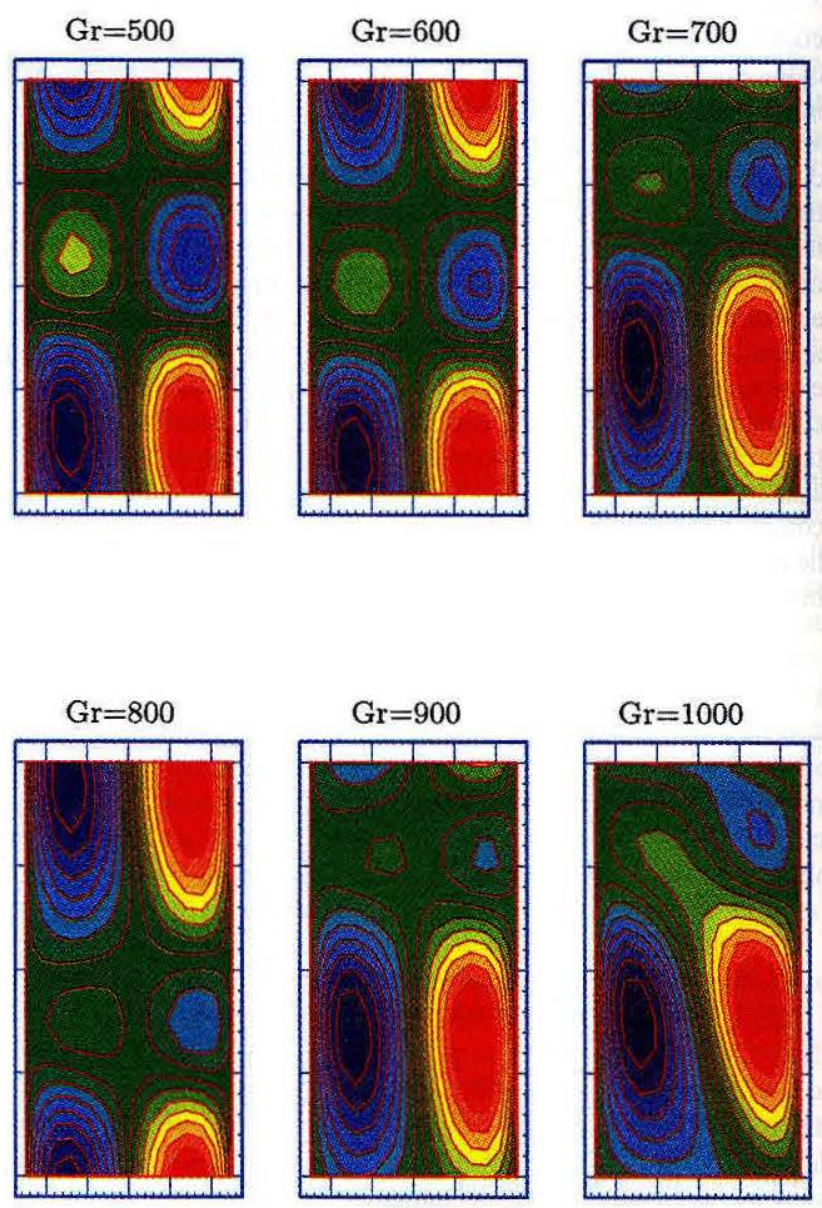

Fig. 2 Change in the size of Taylor vortices with Grashof number for $\eta=$ $0.7, L_{2}=2.001, A=0.67, \mathrm{R}=100$, and $\operatorname{Pr}=0.7$

Gr is increased, a natural convection current is set up. For a positive $\mathrm{Gr}$, the direction of the convection current is upwards near the inner cylinder and downwards near the outer cylinder. As a result of this, the Taylor cell that has the same direction of circulation as the natural convection current increases in size. The counterrotating cell, on the other hand, decreases in size. The counterrotating cell becomes very small for $\mathrm{Gr}=900$, but the flow remains axisymmetric. As $\mathrm{Gr}$ is increased to 1000 , the cell cannot become any smaller and $n=1$ spiral flow is formed. The spiral flow is a Hopf bifurcation of the axisymmetric Taylor vortex flow which occurs between the Grashof numbers of 900 and 1000 . Also 


$$
\begin{aligned}
\frac{\partial u_{\theta}}{\partial t}+\frac{u_{r} u_{\theta}}{r}+u_{r} \frac{\partial u_{\theta}}{\partial r}-\frac{u_{r}}{r} \frac{\partial u_{r}}{\partial \theta}-\frac{u_{z}}{r} \frac{\partial u_{z}}{\partial \theta}+u_{z} \frac{\partial u_{\theta}}{\partial z} & =\frac{1}{r} \frac{\partial P}{\partial \theta}+\frac{1}{R}\left(\frac{1}{r} \frac{\partial u_{\theta}}{\partial r}-\frac{u_{\theta}}{r^{2}}+\frac{\partial^{2} u_{\theta}}{\partial r^{2}}+\frac{1}{r^{2}} \frac{\partial u_{r}}{\partial \theta}\right. \\
& \left.-\frac{1}{r} \frac{\partial^{2} u_{r}}{\partial r \partial \theta}-\frac{1}{r} \frac{\partial^{2} u_{z}}{\partial z \partial \theta}+\frac{\partial^{2} u_{\theta}}{\partial z^{2}}\right)
\end{aligned}
$$

$$
\begin{aligned}
\frac{\partial u_{z}}{\partial t}-u_{r} \frac{\partial u_{r}}{\partial z}+u_{r} \frac{\partial u_{z}}{\partial r}+\frac{u_{\theta}}{r} \frac{\partial u_{z}}{\partial \theta}-u_{\theta} \frac{\partial u_{\theta}}{\partial z} & =-\frac{\partial P}{\partial z} \\
+\frac{1}{R}\left(\frac{1}{r} \frac{\partial u_{z}}{\partial r}-\frac{1}{r} \frac{\partial u_{r}}{\partial z}-\frac{\partial^{2} u_{r}}{\partial r \partial z}+\frac{\partial^{2} u_{z}}{\partial r^{2}}+\right. & \left.\frac{1}{r^{2}} \frac{\partial^{2} u_{z}}{\partial \theta^{2}}-\frac{1}{r} \frac{\partial^{2} u_{\theta}}{\partial z \partial \theta}\right) \\
& +\frac{\mathrm{Gr}}{\operatorname{Re}^{2}}\left(T-\overline{T_{o}}\right)
\end{aligned}
$$
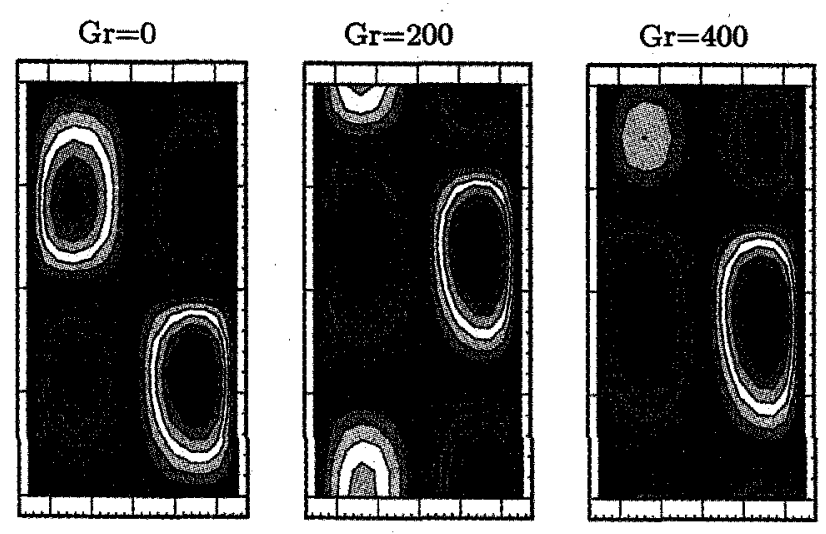

with $\left(u_{r}, u_{\theta}, u_{z}\right)=(0,1,0)$ at the nondimensional inner radius, $\eta /(1-\eta)$ and $\left(u_{r}, u_{\theta}, u_{z}\right)=(0,0,0)$ at the nondimensional outer radius, $1 /(1-\eta)$.

\section{Energy:}

$$
\begin{aligned}
\frac{\partial T}{\partial t}+u_{r} \frac{\partial T}{\partial r}+\frac{u_{\theta}}{r} \frac{\partial T}{\partial \theta}+u_{z} \frac{\partial T}{\partial z} & \\
& =\frac{1}{\operatorname{Pr} R}\left(\frac{1}{r} \frac{\partial T}{\partial r}+\frac{\partial^{2} T}{\partial r^{2}}+\frac{1}{r^{2}} \frac{\partial^{2} T}{\partial \theta^{2}}+\frac{\partial^{2} T}{\partial z^{2}}\right)
\end{aligned}
$$

with $T=1$ at the nondimensional inner radius, $\eta /(1-\eta)$ and $T=$ 0 at the nondimensional outer radius, $1 /(1-\eta)$.

The code was validated by comparing the critical Reynolds number and the corresponding wave speeds with a linear stability analysis that included the effects of radial heating on the centrifugal and gravitational potentials (Chen and Kuo, 1990). In addition, the simulations were also compared with the results of Kataoka et al. (1977) for the local mass transfer coefficient in the limit of small Grashof number, and with the average heat transfer coefficients from the experiments of Ball et al. (1989) for a range of Grashof numbers. Further validations can also be found in Kedia (1997).

It is well known that the axial wavelength depends on Gr. At the outset, it was unclear whether the flow states ( $n=1$ spiral, $n=$ 2 spiral, or aperiodic) would be sensitive to the imposed axial wavelength, $L_{z}$. However, the numerical experiments showed that the flow states were invariant as $L_{z}$ was varied by as much as 30 percent. As $L_{z}$ was varied, the size of the cells were proportionally increased. In the absence of any published results on the exact axial wavelength for nonzero Grashof number and the robustness of the flow states with regard to $L_{2}$, the wavelength for heated flow was chosen as the wavelength at zero Grashof number (widely available from both linear stability analysis and experimental results).

\section{Results}

Flow Transitions and Hysteresis. The variation of the size of the Taylor cells (for a fixed axial wavelength) with the increase in the value of $\mathrm{Gr}$ is shown in Fig. 2. The axial velocity contours (there are two circular contours in the radial direction which corresponds to one Taylor cell) are plotted in the $r-z$ plane for $\eta=$ $0.7, L_{2}=2.001, A=0.67, \mathrm{R}=100$ and $\operatorname{Pr}=0.7$. Note that the critical Reynolds number for the onset of isothermal Taylor cells for a radius ratio of 0.7 is 79.49 . The figure illustrates two important phenomena. The first is the change in the size of Taylor cells and the second is the formation of $n=1$ spiral flow. The spirals move an axial distance of $n L_{z}$ for one rotation around the inner cylinder (Kedia et al., 1998). For $\mathrm{Gr}=0$, there is no effect of natural convection, and both the Taylor cells are of equal size. As
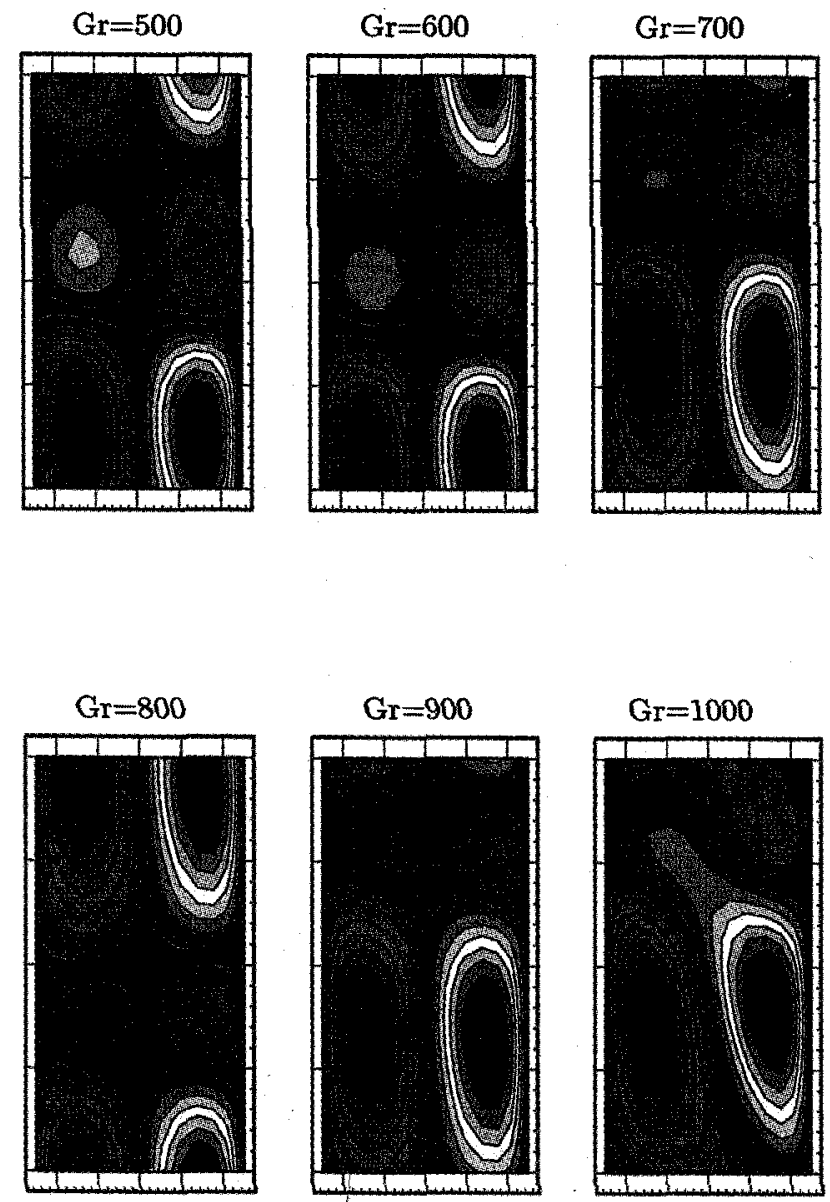

Fig. 2 Change in the size of Taylor vortices with Grashof number for $\eta=$ $0.7, L_{z}=2.001, A=0.67, R=100$, and $\operatorname{Pr}=0.7$

Gr is increased, a natural convection current is set up. For a positive $\mathrm{Gr}$, the direction of the convection current is upwards near the inner cylinder and downwards near the outer cylinder. As a result of this, the Taylor cell that has the same direction of circulation as the natural convection current increases in size. The counterrotating cell, on the other hand, decreases in size. The counterrotating cell becomes very small for $\mathrm{Gr}=900$, but the flow remains axisymmetric. As $\mathrm{Gr}$ is increased to 1000 , the cell cannot become any smaller and $n=1$ spiral flow is formed. The spiral flow is a Hopf bifurcation of the axisymmetric Taylor vortex flow which occurs between the Grashof numbers of 900 and 1000. Also 


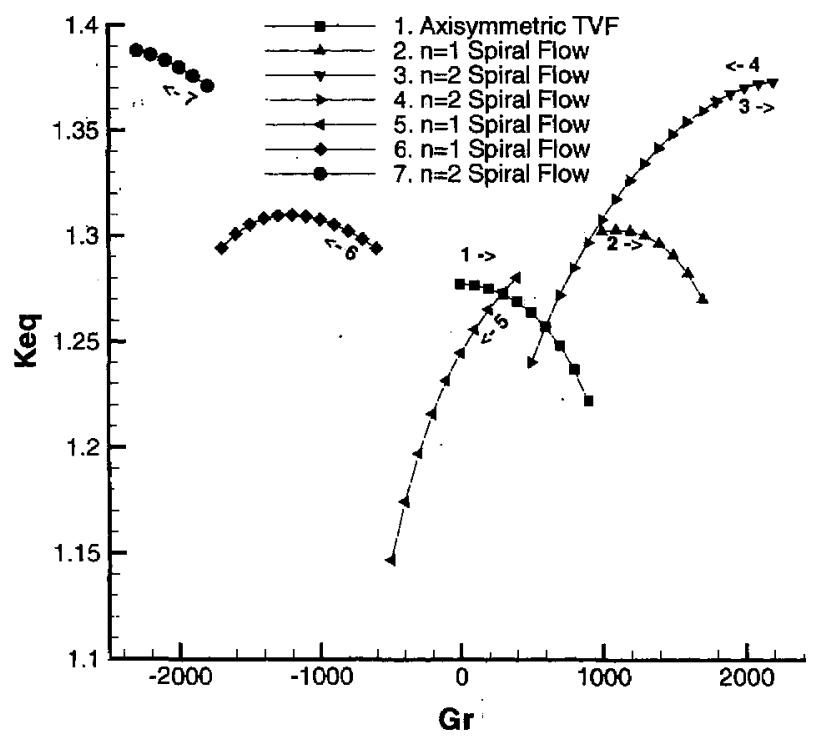

Fig. 3 Map showing different stable states present in the flow by slowly varylng the Grashot numer for $\eta=0.7, L_{z}=2.001, A=0.67, R=100$, and $\operatorname{Pr}=0.7$

the Taylor cells are convected in the axial direction, with the direction depending on the direction of the radial heating. As Gr is increased to 1300 , the flow becomes aperiodic. For negative $\mathrm{Gr}$ (heating of the outer cylinder), Kedia et al. (1998) showed that the flow became aperiodic at higher absolute values of Gr. For $\eta=$ 0.7 , the flow transitioned from axisymmetric to $n=1$ spiral at $\mathrm{Gr}=-900$, and then transitioned to $n=2$ spiral at -1500 . The flow became aperiodic at $\mathrm{Gr}=-2100$. Onset of spiral $(n=1$ and $n=2$ ) flows have also been observed by Kuo and Ball (1997) in certain $\mathrm{Gr}$ and $\mathrm{R}$ parameter space.

The results shown in Fig. 2 were calculated for conditions that would correspond with an impulsive heating and simultaneous acceleration of the cylinder. However, as shown by experimental data, different stable states can be reached by varying the initial conditions of the flow. For example, Coles (1965) observed as many as 25 different stable states at a given cylinder speed for isothermal Taylor-Couette flow. The speed was reached by varying the acceleration rates of the rotating cylinder. The final state in Taylor-Couette flow is thus widely believed to be dependent on its previous history. Figure 3 shows the value of the mean equivalent conductivity and different stable states present in the flow as $\mathrm{Gr}$ is slowly varied. The case is for $\eta=0.7, L_{z}=2.001, A=0.67$, $\operatorname{Pr}=0.7$, and a fixed Reynolds number $(\mathrm{R}=100)$. A random disturbance of magnitude one percent of the velocity of the inner cylinder was introduced to the laminar state (circular Couette flow) and allowed to grow into Taylor vortices. The Reynolds number was slowly increased (in steps of 5) to achieve the desired Reynolds number. The result with the lower Reynolds number was used as initial condition for the next higher Reynolds number. Once the desired Reynolds number was reached, another random disturbance of magnitude one percent of the temperature difference between the two cylinders was imposed on the temperature initial condition in order to trigger transitions. Grashof number was then varied in steps of 100 to achieve the next desired Gr with the previous result used as initial condition. The different states are marked from 1 to 7 in the symbol table in the order in which Gr is slowly increased and then decreased at a constant rotation of the cylinder. As $\mathrm{Gr}$ is slowly increased from 0 to 900 , axisymmetric TVF is formed. From $\mathrm{Gr}=1000$ to $1700, n=1$ spiral flow is formed; $n=2$ spiral flow is formed from $\mathrm{Gr}=1800$ to 2200 . As $\mathrm{Gr}$ is decreased from $2200, n=2$ spiral flow cxists until $\mathrm{Gr}=$ 500. There exist two different stable states (axisymmetric TVF and $n=2$ spiral flow) for $\mathrm{Gr}=600$, but with the same heat transler coefficient. Axisymmetric TVF is achieved by slowly increasing
$\mathrm{Gr}$; then $n=2$ spiral flow is achieved by slowly decreasing $\mathrm{Gr}$. For $\mathrm{Gr}=400$ to $-1700, n=1$ spiral flow is formed. With a further decrease in $\mathrm{Gr}, n=2$ spiral flow is formed for $\mathrm{Gr}=$ -1800 to -2300 . The figure shows the property of nonuniqueness by the existence of a hysteresis loop.

Heat Transfer and Colburn's Analogy. Figure 3 shows the mean cquivalent conductivity, $K_{e 4}=r_{i} \ln (1 / \eta) h_{9, \bar{z},} / k$, as a function of Grashof number for the Grashof numbers steadily increased and then decreased. As shown in the figure. there is a seven percent increase in the average heat transfer coefficient as the flow transitions from an axisymmetric flow to $n=1$ spiral, and an additional eight percent increase in transitioning from a $n=1$ to a $n=2$ spiral. These increases coincide with an increase in the radial velocity component. In the study by Kedia et al. (1998) in which the cylinders wcre impulsively heated, the flow was aperiodic beyond $\mathrm{Gr}=1300$, and the equivalent conductivity was lower, with a value of approximately 1.24 . The previous study also showed that the heat transfer was sensitive to the imposed axial wavelength ( $K_{\text {eq }}$ decreased by 6 percent for a 30 percent incrcase in $L_{f}$ ). Similar variations in the equivalent conductivity occur as the Grashof number is decreased and the flow changes state.

Often it is of interest to compare the variations in the wall shear stress and the effective transport properties for a scalar, as done in the work by Lathrop et al. (1992), for turbulent Taylor-Couette flow. Hence, the results for the average wall shear stress and heat transfer coefficient are presented in terms of the Colburn analogy:

$$
\mathrm{St} \cdot j_{H}(\operatorname{Pr}, \mathrm{Gr}, A, \mathrm{R}, \eta)=C_{f} / 2
$$

where St is the Stanton number, $C_{f}$ is the friction factor, and $j_{H}$ is the Colburn factor, which may be a function of $\operatorname{Pr}, \mathrm{Gr}, A, \mathrm{R}$, and $\eta$. For planar Couette flow, $j_{h}(\operatorname{Pr})=\operatorname{Pr}$, but it is usually equal to $\operatorname{Pr}^{2 / 3}$ for turbulent boundary layers. For circular Couette flow, there is an additional geometric dependence on $\eta$. However, as $\eta$ approaches $1, j_{H}(\mathrm{Pr}, \mathrm{R})=$ Pr. For planar Couette flow and boundary layers, there is no dependence on Reynolds numbers. This invariance with Reynolds numbers was also found for the turbulent Couette flow study by Lathrop et al. (1992).

Figure 4 shows the variation of $j_{H}$ with $\mathrm{R}$ for $\eta=0.7, L_{z}=$ $2.001, \mathrm{Pr}=0.7$, and $\mathrm{Gr}=0$. The geometric factor has been incorporated into $j_{H}$ such that for $\eta=0.7, j_{H}=$ Pr for circular Couette flow. The dots are the simulation results. For $\mathrm{R}=60$ and 70 (circular Couette flow), which are below the critical Reynolds number, $j_{H}=\operatorname{Pr}$. As the Reynolds number is increased, $j_{H}$

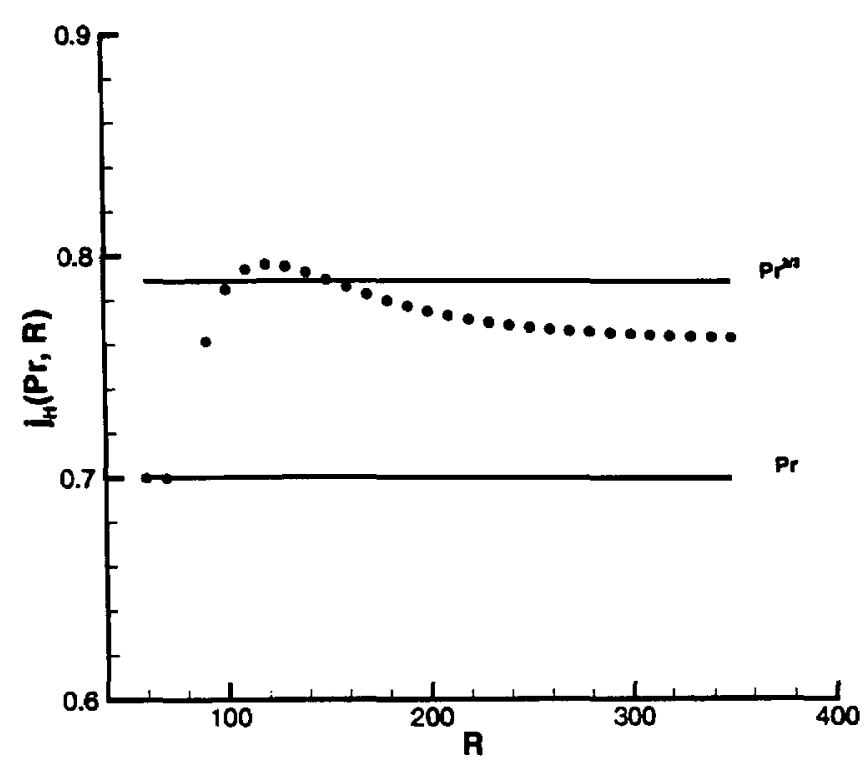

Fig. 4 Variation of $j_{H}(\mathrm{Pr}, \mathrm{R})$ with Reynolds number for $\eta=0.7, L_{z}=$ 2.001, $\mathrm{Pr}=0.7$, and $\mathrm{Gr}=0$ 


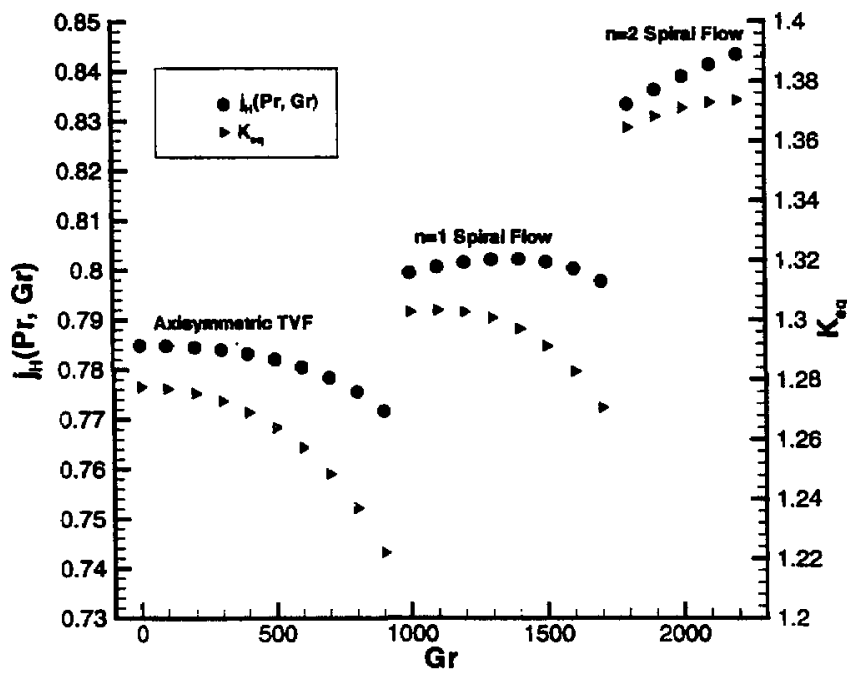

Fig. 5 Variation of $J_{H}(\mathrm{Pr}, \mathrm{Gr})$ and $K_{\mathrm{eq}}$ with Grashof number for $\eta=0.7$, $L_{z}=2.001, A=0.67, \mathrm{Pr}=0.7$, and $R=100$

increases and reaches a maximum just above $\operatorname{Pr}^{2 / 3}$ and finally becomes constant at $0.76=\operatorname{Pr}^{0.77}$. Hence, just beyond the critical Reynolds number, the Colburn factor does depend on the Reynolds number, but the dependence decreases for higher Reynolds numbers. Figure 5 shows a similar correlation for $\eta=0.7, L_{z}=$ 2.001, $A=0.67, \operatorname{Pr}=0.7$, and $\mathrm{R}=100$. Here $j_{H}(\mathrm{Pr}, \mathrm{Gr})$ is plotted with $\mathrm{Gr}$ for a fixed Reynolds number $(\mathrm{R}=100)$. At the Grashof numbers corresponding to changes from axisymmetric to $n=1$ spiral flow, and from $n=1$ spiral to $n=2$ spiral, both the friction factor and the equivalent conductivity exhibit similar increases in value.

Time-Delay Analysis. To characterize the flow as the Grashof number is changed, an analysis of the temporal variations of radial velocity and heat transfer coefficient is performed. The radial velocity halfway between the inner and the outer cylinders at the mid-axial position is recorded at every time step. Figure 6 shows the fluctuating component of the radial velocity (the mean velocity has been subtracted) for $\eta=0.7, L_{z}=2.001, \mathrm{R}=100, \mathrm{Pr}=0.7$, and $\mathrm{Gr}=-1700$. The time is nondimensionalized using the time period of the inner rotating cylinder. The flow is in the $n=2$ spiral flow regime. The time trace looks regular and periodic. Shown in Fig. 7 is a similar velocity trace for $\mathrm{Gr}=-2100$. The time trace has become irregular and the flow is in the aperiodic regime. Both

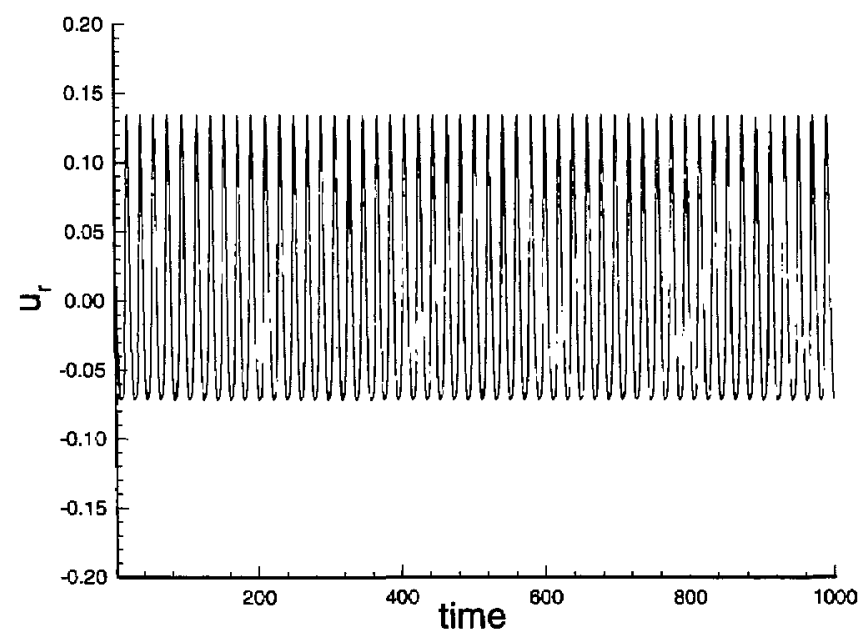

Fig. 6 Radial velocity signal for $\eta=0.7, L_{z}=2.001, \mathrm{R}=100, \mathrm{Pr}=0.7$, and $\mathrm{Gr}=-1700$

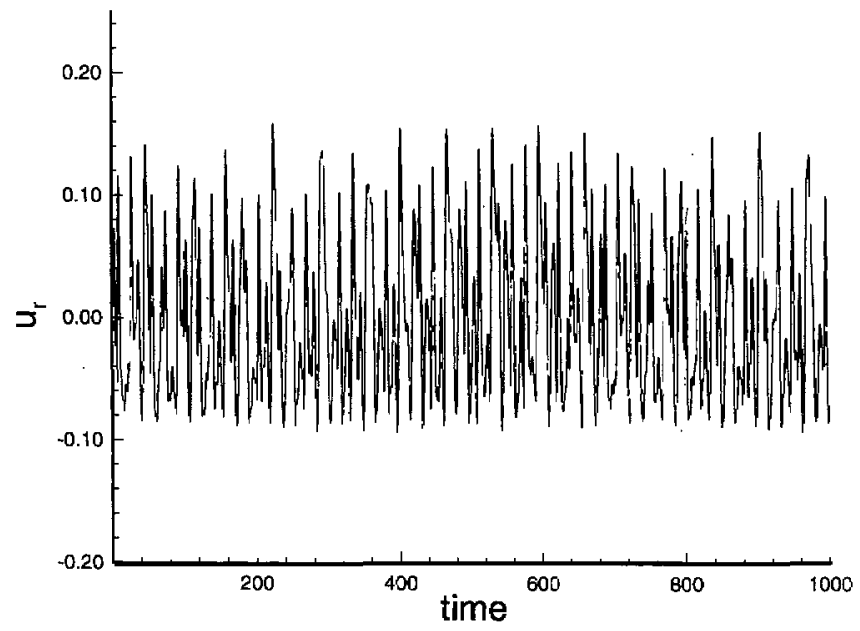

Fig. 7 Radial velocity signal for $\eta=0.7, L_{z}=2.001, \mathrm{~B}=100, \mathrm{Pr}=0.7$, and $\mathrm{Gr}=-2100$

the time traces are shown after the initial transients have died down. The power spectra of the radial velocity time series were obtained by performing a fast Fourier transform. Shown in Figs. 8

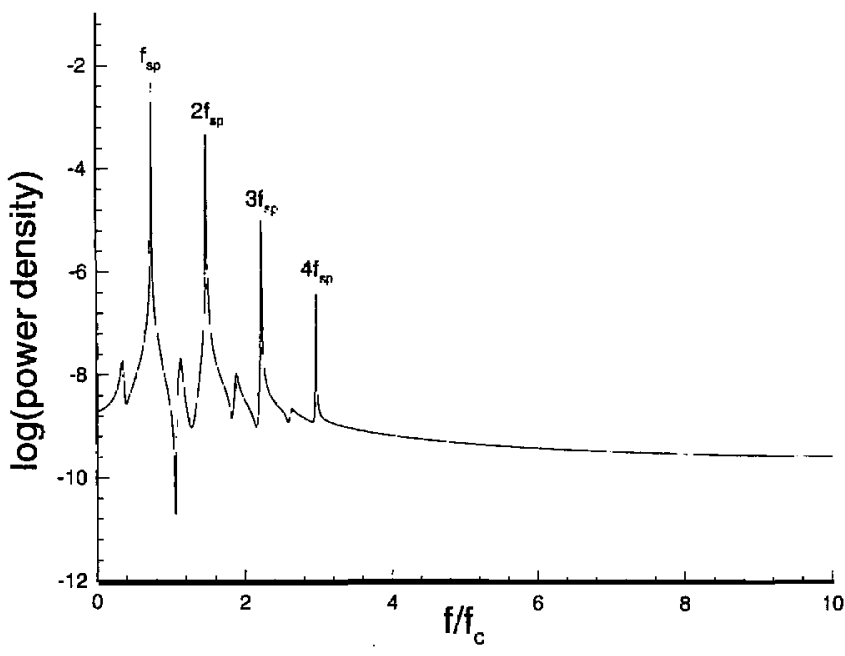

Fig. 8 Power spectrum of the radial velocity for $\eta=0.7, L_{x}=2.001, A=$ $0.67, R=100, \operatorname{Pr}=0.7$, and $\mathrm{Gr}=-1700$

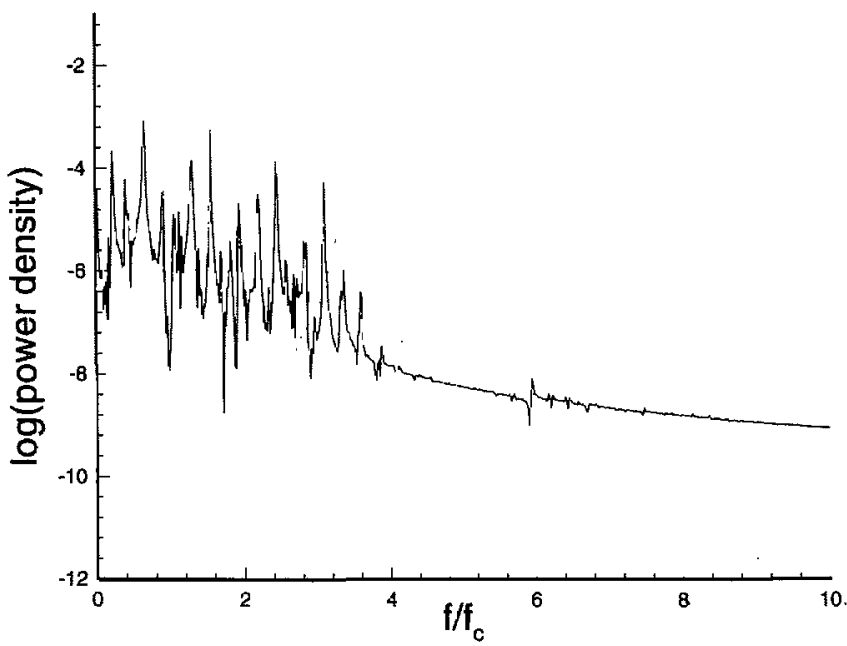

Fig. 9 Power spectrum of the radial velocity for $\eta=0.7, L_{z}=2.001, A=$ $0.67, \mathrm{R}=100, \mathrm{Pr}=0.7$, and $\mathrm{Gr}=-2100$ 


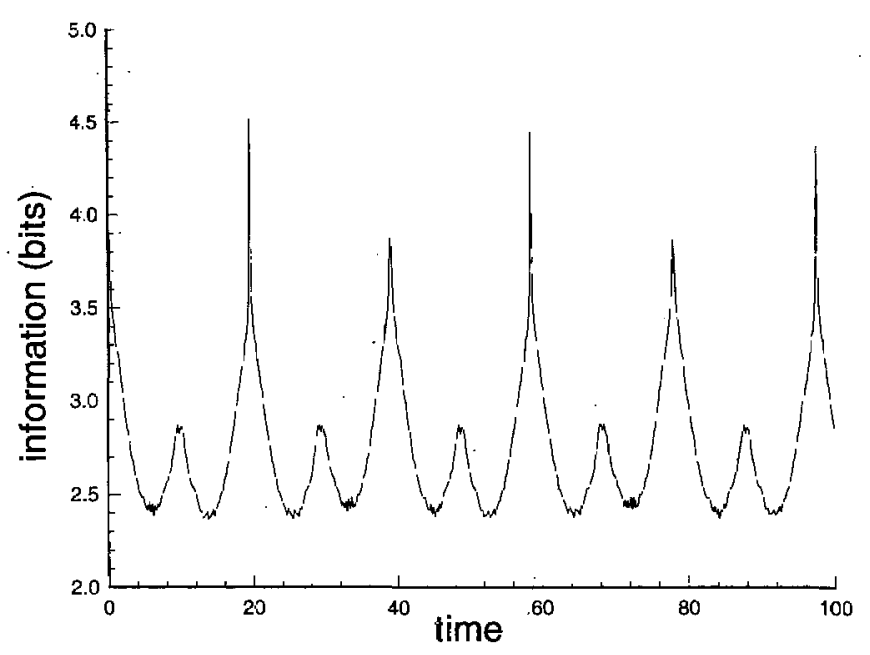

Fig. 10 Mutual information of the radial velocity for $\eta=0.7, L_{x}=2.001$, $A=0.67, \mathrm{~A}=100, \mathrm{Pr}=0.7$, and $\mathrm{Gr}=-1700$

and 9 are the respective power spectra for $\mathrm{Gr}=-1700(n=2$ spiral flow) and $\mathrm{Gr}=-2100$ (aperiodic flow). Figure 8 shows a fundamental frequency at $f / f_{c}=0.74$ and its harmonics. Here, the frequency is nondimensionalized using the inner cylinder frequency $\left(f_{c}\right)$. The flow is clcarly periodic in nature. For the case of $\mathrm{Gr}=-2100$ (Fig. 9), the peaks for $\mathrm{Gr}=-1700$ are still present but their amplitudes have slightly decreased. Also, other frequencies have appeared in the flow that are of comparable power density.

For the broadband spectra at $\mathrm{Gr}=-2100$, the flow may be rcpresented as a low dimensional dynamical system using timedelay coordinates to reconstruct the phase space (Takens, 1981) To calculate the dimension of the attractor, a suitable time delay must be determined. For this purpose, the mutual information function, $J(\tau)$, is used (Fraser and Swinney, 1986)

$$
\Lambda(\tau)=\iint P(X, Y) \log _{2}[P(X, Y) / P(X) P(Y)] d X d Y
$$

where $X=v(t)$ and $Y=v(t+\tau) ; P(X)$ and $P(X, Y)$ are the probability density and the joint probability density and $\tau$ is a suitable time delay. Given the value of $v(t)$, the mutual information function gives the accuracy with which $v(t+\tau)$ can be predicted. Since $v(t)$ and $v(t+\tau)$ denote the coordinates of the

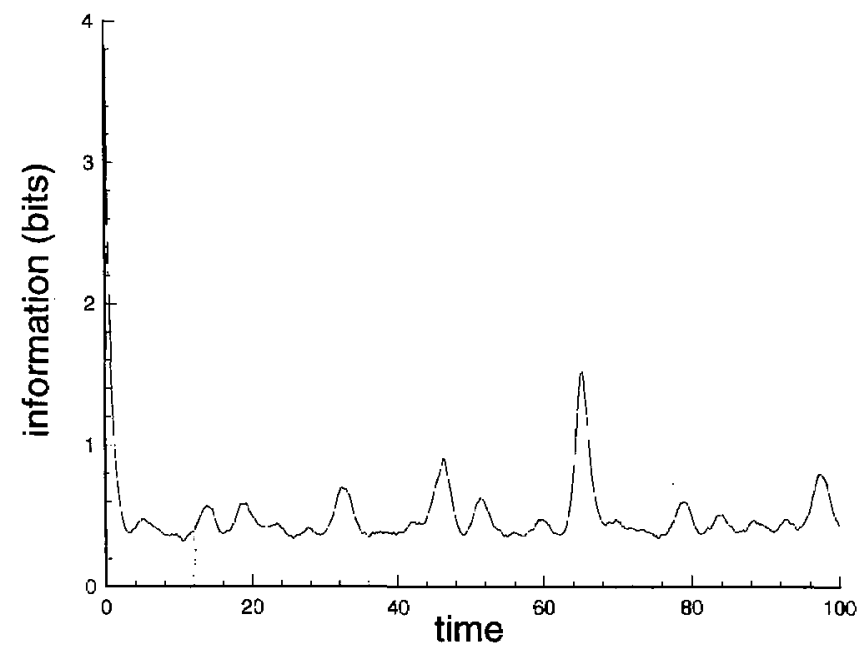

Fig. 11 Mutual information of the radial velocity for $h=0.7, L_{z}=2.001$, $A=0.67, \mathrm{R}=100, \mathrm{Pr}=0.7$, and $\mathrm{Gr}=-2100$

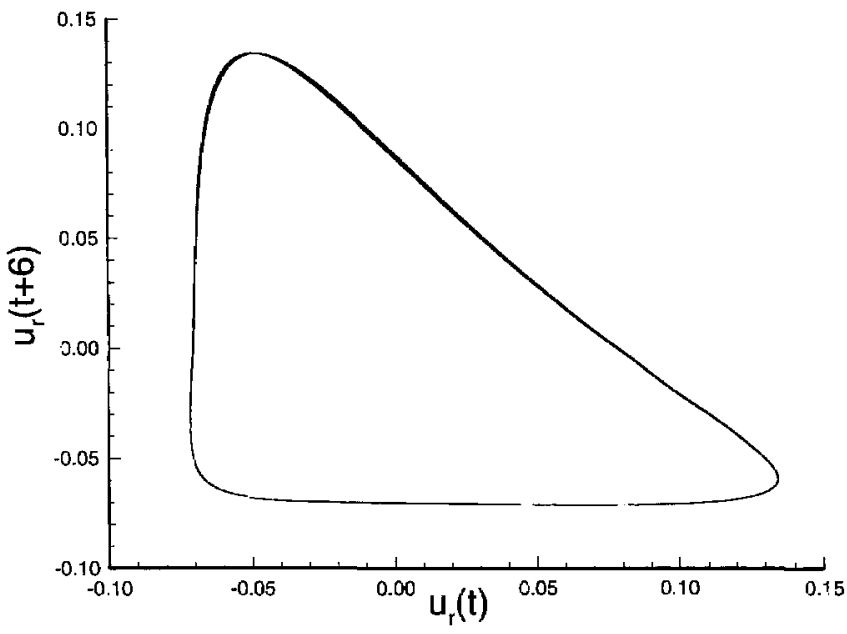

Fig. 12 Phase plot of the radial velocity for $\eta=0.7, L_{x}=2.001, A=0.67$ $\mathrm{R}=100, \mathrm{Pr}=0.7$, and $\mathrm{Gr}=-1700$

reconstructed phase space, they should be independent of each other. Therefore, a good choice for the time delay is the first local minimum (Fraser et al., 1986) of the mutual information. The mutual information function is shown for $\mathrm{Gr}=-1700$ and $\mathrm{Gr}=$ -2100 in Figs. 10 and 11 , and the first local minimums occur at $t=6$ and $t=3$, respectively. Note that for $\mathrm{Gr}=-1700$, the mutual information function is periodic as expected for a periodic signal.

The phase plots for $\mathrm{Gr}=-1700$ and $\mathrm{Gr}=-2100$ are shown in Figs. 12 and 13. For $\mathrm{Gr}=-1700$, a clcan limit cycle is seen for $n=2$ spiral flow (periodic). For $\mathrm{Gr}=-2100$, the reconstructed attractor has become vastly irrcgular and the limit cycle has disappeared.

In the present study both the pointwise dimension, $D_{1}$, and the correlation dimension, $D_{2}$, are calculated using the following definitions (Farmer et al., 1983):

$$
D_{1}=\lim _{\epsilon \rightarrow 0} \frac{\langle\log N(\epsilon)\rangle_{x}}{\log \epsilon}
$$

where $N(\epsilon)$ is the number of points in a hypersphere of radius $\epsilon$. The averaging is done over different referencing points $x$. The correlation dimension is similar to the pointwise dimension but the averaging is done over the number of points $N(\epsilon)$,

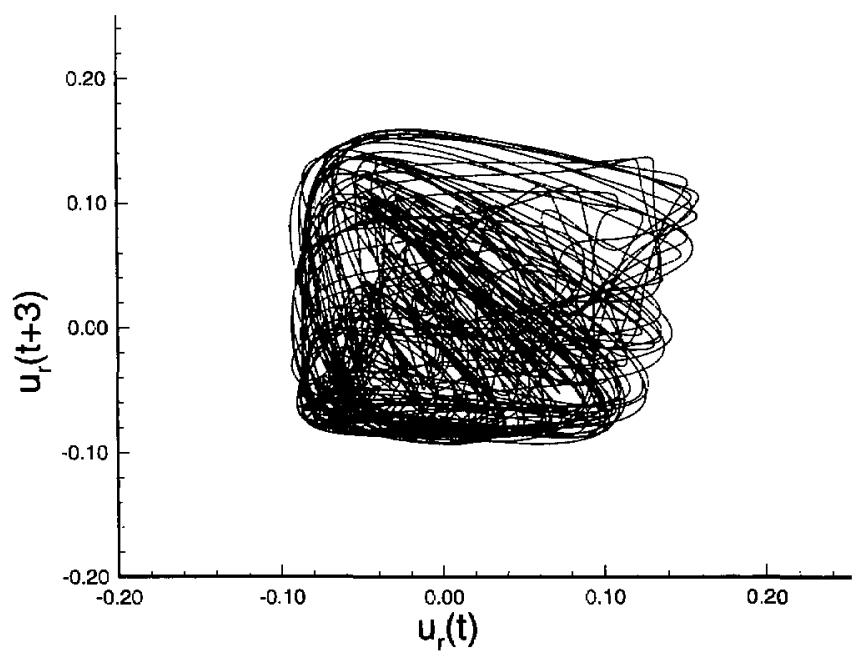

Fig. 13 Phase plot of the radial velocity for $\eta=0.7, L_{z}=2.001, A=0.67$, $\mathrm{R}=100, \mathrm{Pr}=0.7$, and $\mathrm{Gr}=-2100$ 


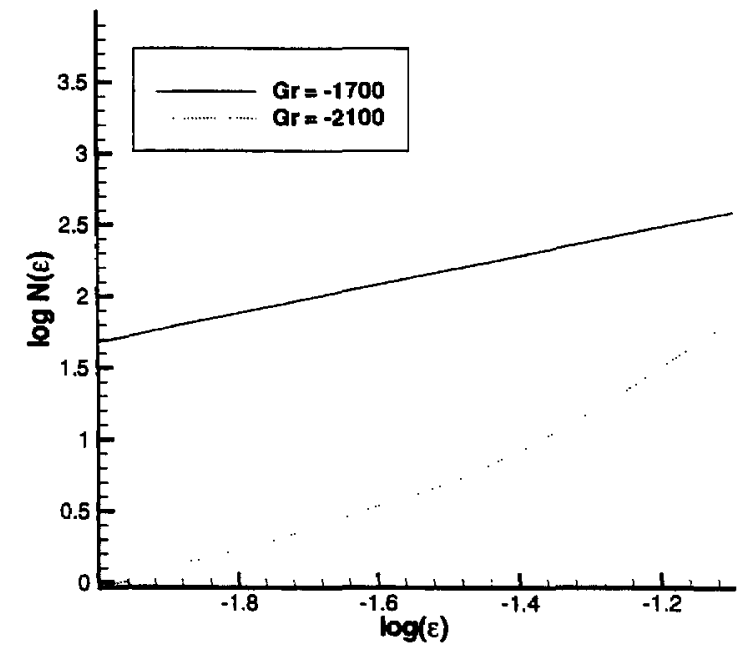

Fig. 14 Plot of $\log M(\epsilon)$ versus $\log (\epsilon)$ from the radial velocity for $\eta=0.7$, $L_{z}=2.001, A=0.67, R=100$, and $\operatorname{Pr}=0.7$

$$
D_{2}=\lim _{\epsilon \rightarrow 0} \frac{\log \left\langle N(\epsilon\rangle_{x}\right.}{\log \epsilon}
$$

Both the pointwise dimension $D_{1}$ and the correlation dimension $\mathrm{D}_{2}$ were computed using the same algorithms, once by averaging the logarithm of the number of points and again by taking the logarithm of the averaged points. There was no appreciable change in these two calculated dimensions. Therefore, only $D_{1}$ is reported here. Embedding was done in six-dimensional phase space. Higher dimensional embedding was also done with no change in the attractor dimension. For a six-dimensional embedding, 256 reference points were chosen. These reference points were selected using uniformly generated random numbers, so that the reference points are spread out through the entire attractor equally. The pointwise dimension $D_{1}$ is shown for the two different flows ( $n=$ 2 spiral flow and aperiodic flow). Plots of $\log N(\epsilon)$ versus $\log (\epsilon)$ for $\mathrm{Gr}=-1700$ and $\mathrm{Gr}=-2100$ are shown in Fig. 14. The slopes of the graphs give the dimension of the reconstructed attractor. The slopes for the above cases are shown in Figs. 15 and 16. For $\mathrm{Gr}=$ -1700 , a constant slope of approximately 1 is seen which reflects the periodic nature of the reconstructed attractor. For $\mathrm{Gr}=-2100$ (Fig. 16), a slope of about 3.2 in the $\log (\epsilon)$ interval of $(-1.3$, $-1.1)$ is visible. The attractor dimension reaches an asymptotic

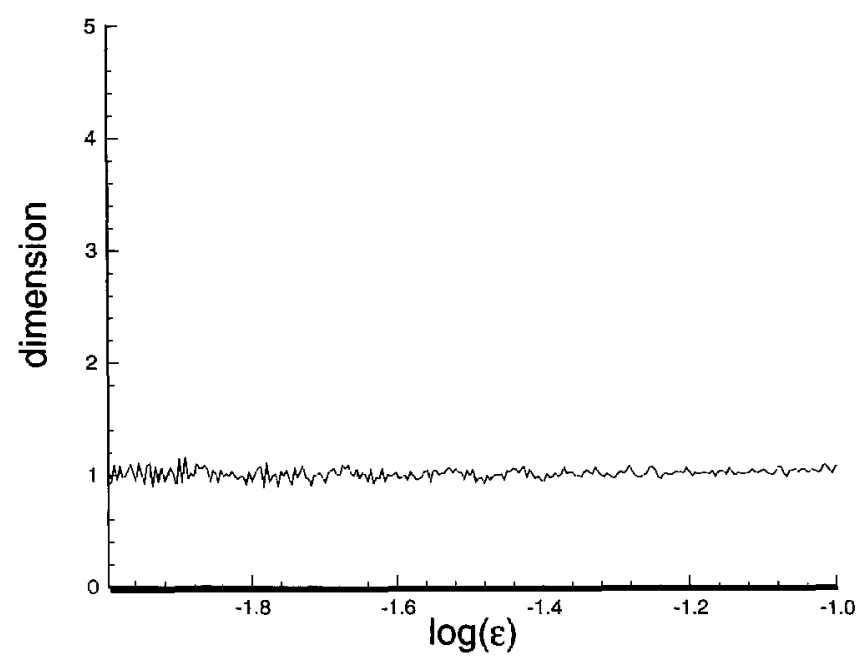

Fig. 15 Plot of the slope from the radial velocity for $\eta=0.7, L_{z}=2.001$, $A=0.67, \mathrm{H}=100, \operatorname{Pr}=0.7$, and $\mathrm{Gr}=-1700$

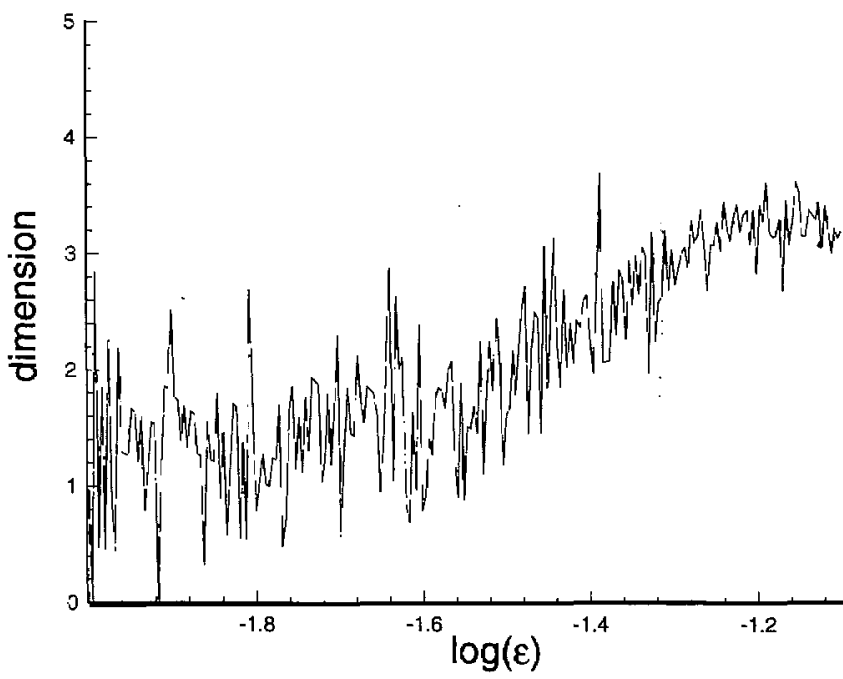

Fig. 16 Plot of the slope from the radial velocity for $\eta=0.7, L_{2}=2.001$, $A=0.67, \mathrm{R}=100, \mathrm{Pr}=0.7$, and $\mathrm{Gr}=-2100$

value as the embedding dimension is increased (Table 1). If three irrationally related frequencies exist in the flow, the dimension of the flow would be 3 .

The same process of time delay analysis is repeated for the local heat transfer coefficient $(h)$ at one location within the flow. The time series for the local heat transfer coefficient and the radial velocity look very similar. The power spectra for the two Grashof numbers are given in Figs. 17 and 18. The magnitude of the power is two orders of magnitude higher than the corresponding power spectra for the radial velocity. However, the peaks occur at virtually the same frequencies. Shown in Figs. 19 and 20 are the dimension calculations for the local coefficients of heat transfer.

Table 1 Pointwise dimension as a function of the embedding dimension

\begin{tabular}{cc}
\hline$m$ & $D_{1}$ \\
\hline 3 & $2.50 \pm 0.1$ \\
4 & $3.00 \pm 0.1$ \\
5 & $3.15 \pm 0.1$ \\
6 & $3.20 \pm 0.1$ \\
7 & $3.20 \pm 0.1$ \\
8 & $3.20 \pm 0.1$ \\
\hline
\end{tabular}

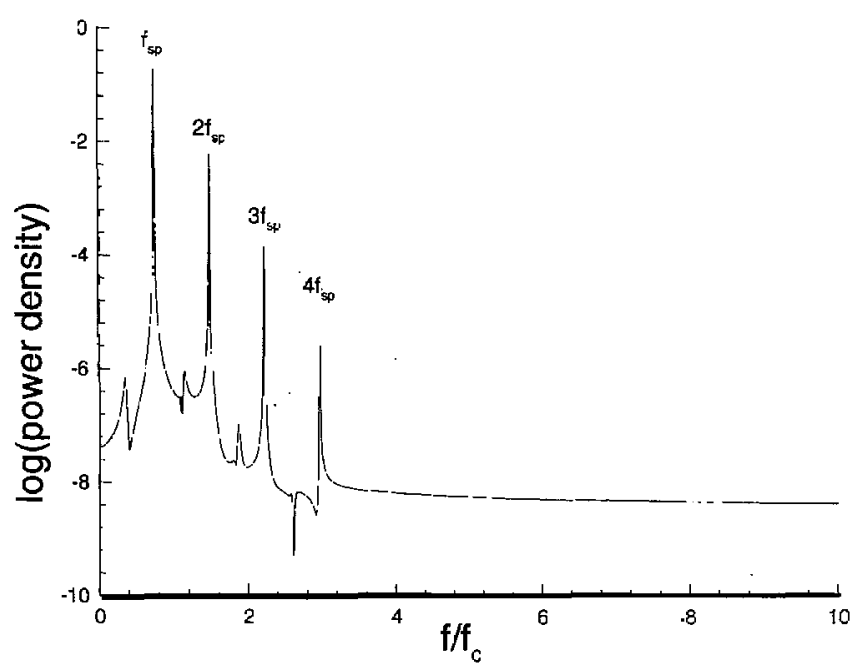

Fig. 17 Power spectrum of the local heat transfer coefficient for $\eta=0.7$, $L_{z}=2.001, A=0.67, \mathrm{R}-100, \mathrm{Pr}=0.7$, and $\mathrm{Gr}=-1700$ 


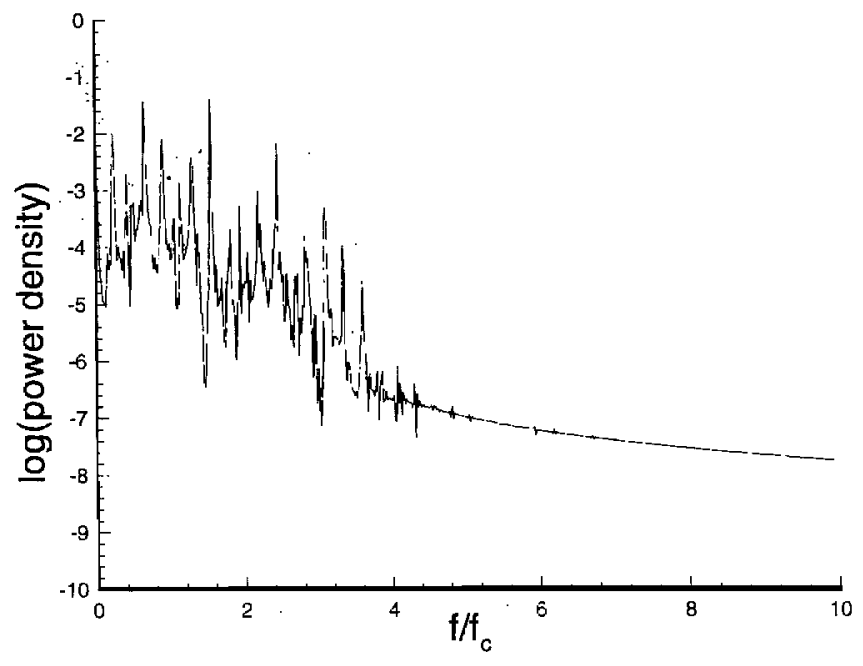

Fig. 18 Power spectrum of the local heat transfer coefficient for $\boldsymbol{\eta}=\mathbf{0 . 7}$, $L_{z}=2.001, A=0.67, R=100, P r=0.7$, and $\mathrm{Gr}=-2100$

The dimension for $\mathrm{Gr}=-2100$ is approximately 3.2 , which agrees well with the dimension obtained from the radial velocity time trace. Therefore, it is concluded that the local heat transfer coefficient follows the radial velocity pattern, with the reason being the active role of the radial velocity component in transferring heat between the two cylinders. The information about the phase space, such as the temporal evolution of the trajectories and the dimension of the attractor, can be used for making models such as predicting the value of the heat transfer coefficient for aperiodic flow. These models can then be used for predicting future behavior of the dynamical system, i.e., extracting the value of $v(t+1)$ given a long time series of $v(t)$.

\section{Conclusion}

Heat transfer in an incompressible three-dimensional TaylorCouette flow with a rotating inner cylinder has been numerically investigated to study the interaction of gravity and centrifugal potentials with the radial temperature gradient. Both the aforementioned effects have not been considered together in previous numerical simulations. For a fixed axial wavelength, the individual size of the Taylor vortex pair changes with the Grashof number,

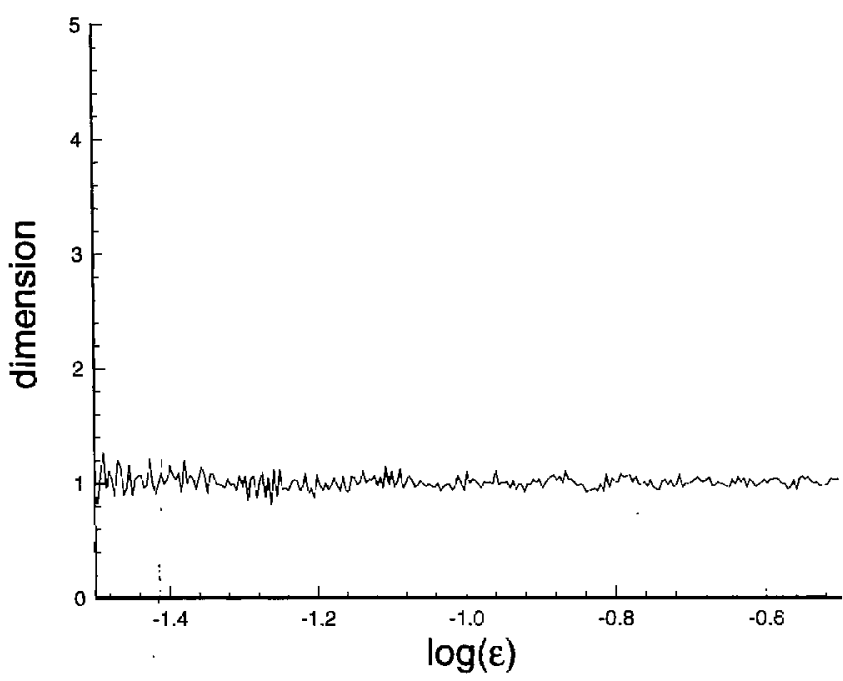

Fig. 19 Plot of the slope from the local heat transfer coefficient for $\eta=$ $0.7, L_{x}=2.001, A=0.67, \operatorname{R}=100, \operatorname{Pr}=0.7$, and $\mathrm{Gr}=-1700$

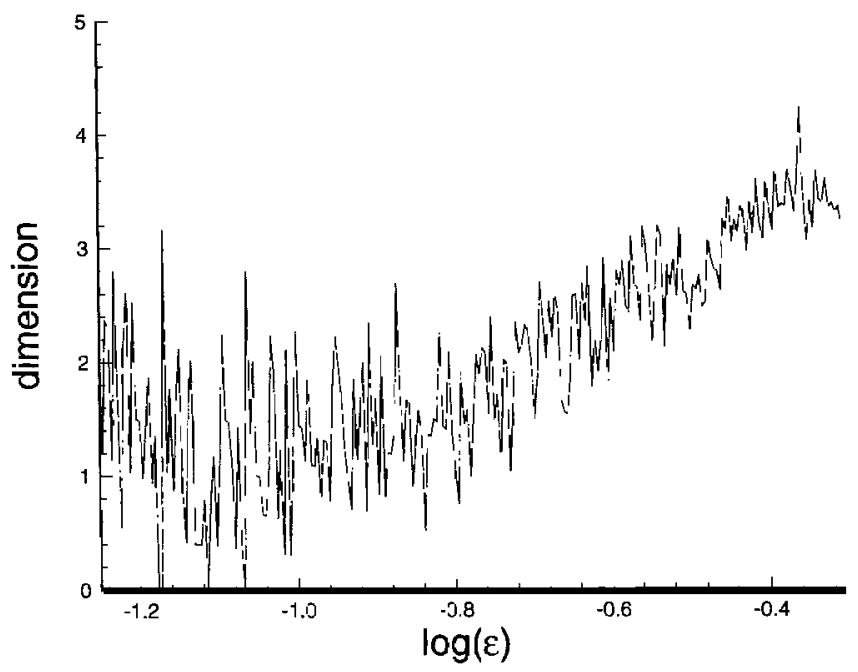

Fig. 20 Plot of the slope from the local heat transter coefficient for $\eta=$ $0.7, L_{z}=2.001, A=0.67, \mathrm{~B}=100, \mathrm{Pr}=0.7$, and $\mathrm{Gr}=-2100$

corresponding to a transition to a spiral flow regime with a significant increase in the average heat transfer.

One of the interesting properties of the nonlinear Navier-Stokes equations is nonuniqueness. With all other parameters fixed, the Grashof number was slowly varied to examine the existence of a hysteresis loop in heated Taylor-Couette flow. It was found that two stable states cxisted for the same Grashof number. One state was reached by increasing the Grashof number and the other by slowly decreasing it.

The validity of Colburn's correlation was also investigated to indicate the coupling between the wall stress and the transport of a scalar. The function $j_{H}(\mathrm{Pr}, \mathrm{R})$ was found to be slightly lower than $\operatorname{Pr}^{2 / 3}$ (note that the exponent is 0.77 . which is higher than $\frac{2}{3}$ ) lor the range of Reynolds number numerically investigated and to be dependent on the Reynolds number near the critical speed. For a fixed Reynolds number, the friction factor and the equivalent conductivity followed similar trends with Grashof number.

The frequency power spectrum for $n=2$ spiral flow showed the single fundamental frequency and its harmonics for $\mathrm{Gr}=-1700$. For $\mathrm{Gr}=-2100$, new frequencies appeared in the power spectrum. The power spectrum became broadbanded for even lower Grashof numbers. The two-dimensional projection of the reconstructed attractor showed a limit cycle for $\mathrm{Gr}=-1700$. The limit cycle behavior disappeared at $\mathrm{Gr}=-2100$ and the reconstructed attractor became irregular. The pointwise dimension calculation was approximately $\mathrm{I}$ for $\mathrm{Gr}=-1700$. The dimension value incrcased to about 3.2 for $\mathrm{Gr}=-2100$ and the constant dimension region was found to be small. These dimensions were found from the trace of the radial velocity, and from the variation in the local heat transler. Hence, the dynamics of the heat transfer variations can be modelled exactly the same as that of the radial velocity, which may be easier to assess in certain physical arrangements.

\section{Acknowledgments}

This research was performed in part using the CSCC parallel computer system operated by Caltech on behalf of the Concurrent Supercomputing Consortium. Access to this facility was provided by Caltech. The authors would also like to thank Dr. A. Leonard at Caltech and Dr. R. D. Moser at University of Illinois at UrbanaChampagne for valuable discussions.

\section{References}

Ball, K. S., Farouk, B.. and Dixit, V. C., 1989. "An experimental study of heat transfer in a vertical annulus with a rotating innet cylinder." Int. J. Heal Mas. Transfer, Vol. 32, No. 8, pp. 1517-1527. 
Brandstater, A., and Swinney, H. L., 1987, "Strange attractors in weakly turbulent Couctic-"laylor flow," Phys. Rev. A, Vol. 35, No. 5, pp. 2207-2220.

Chen, J., and Kuo, J., 1990, "The lincar stability of stcady circular Couette flow with a small radial temperalure gradient," Phys. Fluids A, Vol. 2, No. 9, pp. 1585-1591.

Coles, D., 1965, "Transition in circular Couete flow," J. Fluid Mech., Vol. 21, pp. $385-425$.

Farmer. J. Doync, OLL, E., and Yorke, J. A., 1983, "The dimension of chaotic atractors," Physica $7 D$. pp. 153-180.

Fraser, A. M., and Swinney, H. I., 1986, "Independent coordinates for strange attractors from mutual information," Physical Review' A, Vol. 33, No. 2, pp. 11341140.

Kataoka, K., Doi, H., and Komui, T., 1977, "lleal/Mass transter in Taylor vortex flow with constant axial flow rates," Int. J. Heat Mass Transfer, Vol. 20, pp. 57-63.

Kedia, R., 1997, "An investigation of velocity and temperature ficlds in TaylorCuuette fluws," Ph.1), thesis. California Institute of lechnology, Pasadena, CA.

Kedia, R., Hunt, M. L., and Colonius. T., 1998, "Numerical Sinulations of Heat
Transfer in Taylor-Couette Flows," ASME Joursal of llFat TKansfer, Vol. 120, pp. $65-71$.

Koschmieder, E. I.., 1993. Benard Catls and Tayior Vortices, Cambridge University Fross, New York.

Kuo, D. C., and Ball, K. S., 1997, "Taylor-Coucte flow with buoyancy: Onsel of spiral fiow," Phys. Fluids A, Vol. 9, No. 10, pp. 2872-2884.

[athrop, D. P., Fineberg, J., and Swinney, H. L., 1992, "Turbulent flow between concentric rotating cylinders at large Reynolds number," Physical Review Letters, Vol. 68. No. 10, pp. 1515-1518.

Narabayashi, T., Miyano, H., Komita, H., likurd. T., Shiina, K.; Kalo. H.. Watanabe, A.. and 'l'akubashi, Y., 1993, "Study on temperature fluctuation mechanisms in an annulus gap hetween PI,R Pump shatt and casing cover," Proceedings of the Se'end ASME/JSME Joint Conference an Nuciear Engincering, P. F. Peterson. ed. Mar. 21-24, San Francisco, CA, ASME, New York.

Takens, F., 1981, "Detecting strange attractors in turbulence," lecture Notes in Mathematics, 898, D. Rand and L. S. Young, eds., Springer, Berlin, pp. $366-381$.

Taylor, G. I., 1923. "Stability of a viscous liquid contained between two rolating cylinders," Phils. Trans. R. Soc. London, Vol. A223, pp. 289-343.

\title{
E R R A T A
}

The 1998-1999 Heat Transfer Division Exccutivc Committee should have been listed on the Journal masthead (August 1998 through May 1999 issues) as follows:

\author{
Chair, W. A. Fiveland \\ Vice Chair, R. A. Nelson \\ Member, I. C. Witte \\ Secretary, J. H. Kim \\ Treasurer, Y. Jaluria \\ Past Chair, G. P. Peterson
}

